\title{
Cystic Fibrosis-Related Diabetes
}

\author{
Bernadette Prentice, Shihab Hameed, Chee Y. Ooi, \\ Charles F. Verge and John Widger
}

Additional information is available at the end of the chapter

http://dx.doi.org/10.5772/66452

\begin{abstract}
Cystic fibrosis-related diabetes (CFRD) results in significant morbidity and mortality for patients with cystic fibrosis (CF). It is the endpoint of a spectrum of progressive insulin deficiency with resulting abnormalities of glucose tolerance. The consequence of glycaemic abnormalities in CF is poorer nutritional status, an increase in respiratory exacerbations with decline in lung function and ultimately greater morbidity and mortality. CFRD can be diagnosed by the standard oral glucose tolerance test (OGTT) usually performed from 10 years of age. However, this may miss early glycaemic abnormalities which appear to be clinically important. Early recognition of CFRD and treatment have been shown to improve outcomes in CF. Novel diagnostic methods such as 30-min sampled OGTT and continuous glucose monitoring (CGM) may prove to be useful in screening for this disorder and in the early identification of glycaemic abnormalities.
\end{abstract}

Keywords: cystic fibrosis-related diabetes, glucose, insulin, abnormal glucose tolerance, indeterminate glycaemia, impaired glucose tolerance, oral glucose tolerance test, continuous glucose monitoring

\section{Introduction}

Cystic fibrosis (CF) is the most common life-limiting autosomal recessive genetic condition seen in the Caucasian population, affecting approximately 1/2500 live births in Australia [1]. It is caused by mutations in the cystic fibrosis transmembrane regulator (CFTR) gene, located on the long arm of chromosome 7 [1] and expressed in the epithelial cells of lungs, pancreas and sweat glands and other organs. Cystic fibrosis-related diabetes (CFRD) is one of the most important complications of the disease as it is known to have a significant impact on morbidity and mortality [2]. Patients with CF ultimately die from recurrent respiratory tract infections 
and respiratory failure which may be hastened by abnormalities of glucose tolerance affecting respiratory function and nutrition.

\section{Pathophysiology}

The pathophysiology of CFRD is likely multifactorial and complex. Historically CFRD was thought to be the result of progressive pancreatic destruction by secretions of the exocrine pancreas, pancreatic autodigestion and replacement with nonfunctioning fatty tissue, amyloid deposits or fibrotic tissue [3,4]. This theory was supported by ultrasound findings in patients with CF of an "echogenic" and atrophied pancreas which progresses with age. MRI has also been used to study the pancreas of patients with CF. Sequeiros et al. attempted to determine the pancreatic volume of patients with CF using MRI and compare with Type 1 diabetic patients and controls. In over $70 \%$ of patients with $\mathrm{CF}$, the pancreas could not be visualised and this was irrespective of glycaemic status [5]. Pancreatic tissue on autopsies of patients with CF has also noted to have fewer islet cells and replacement with fibrotic tissue. Histologically, patients with CFRD have a relative decrease in the number of islet cells and insulin-containing cells within the islets, relative to the non-CFRD cohort $[4,6]$.

However, recent information supports the theory that destruction of the physical pancreas does not entirely explain the glycaemic abnormalities in patients with CF. Insulin deficiency has been shown to occur in young children and infants with CF [7], and even infants have been reported to have CFRD [8]. This has also been demonstrated in animal models of CF. In both the pig and ferret CF models, the animals demonstrate abnormal insulin secretion from birth, suggesting that CFTR may play a more direct role in insulin secretion $[9,10]$. In the pig model, newborn pigs were noted to develop hyperglycaemia even when there was no significant islet cell destruction [10]. Recent studies of the CFTR potentiator ivacaftor $\left(\right.$ Kalydeco $\left.^{\mathrm{TM}}\right)$, which improves gating defects and thus should not have any impact on fatty or fibrotic tissue, have demonstrated an improvement in glucose abnormalities [11, 12]. This suggests that the intrinsic abnormality in the CFTR protein may play a role in glycaemic control in CF.

The timeframe during which patients with CF develop glycaemic abnormalities and CFRD has significant variability, and the specific CFTR class abnormality does not entirely account for this unpredictability. Non-CFTR genetic modifiers may play a key role in determining this risk. Patients with CF who have a family history of Type 2 diabetes are known to have an increased risk of CFRD [13, 14]. Polymorphisms in TCF7L2, a "susceptibility gene" for Type 2 diabetes, are more common in patients with CFRD. The pathophysiology also appears to have similarities. Couce et al. noted that islet cell amyloidosis, which is characteristic of pancreatic histology of patients with Type 2 Diabetes mellitus, is also present in CF patients with CFRD and "borderline diabetes" but not in nondiabetic CF patients or controls [3]. Other genetic modifiers have been shown to modify CF phenotypes, including SLC26A9 which has been demonstrated to be more common in patients who develop CFRD [15].

In normal insulin physiology, insulin secretion occurs in two phases - the first phase results from exocytosis of preformed insulin granules which is the result of a voltage-dependent 
calcium channel being triggered by blood glucose elevations [16-18]. The second phase requires maturation of insulin granules and lasts minutes to hours [19, 20]. Oral glucose ingestion results in a limited and delayed first-phase insulin peak when compared with intravenous administration [21, 22]. Overall, the amount of insulin secreted appears to be amplified when glucose is given orally, rather than intravenously. Incretins (glucagon-like peptide and gastric inhibitory peptide) are secreted from neuroendocrine cells of the gastrointestinal system and increase insulin secretion and decrease glucagon secretion. The secretion of incretins is hypothesised to be the result of the action of oral glucose within the gastrointestinal tract [21]. The role of incretins in CFRD has not yet been fully elucidated, and it is unclear whether or not patients with CFRD have abnormal levels of incretins. However, the diet of CF patients may play a role in the development of CFRD. In patients with Type 2 diabetes mellitus (DM), those prescribed orlistat, a lipase inhibitor, had diminished fat digestion which resulted in greater postprandial hyperglycaemia [23]. In a randomised crossover trial, Perano et al. demonstrated that adolescent patients with CF, who did not take appropriate pancreatic enzyme supplementation, experienced amplified postprandial hyperglycaemia [24]. Barrio postulates in her review that inadequate enzyme supplementation in patients with CF results in fat malabsorption, which may hasten gastric emptying, thereby inhibiting the normal augmentation of insulin response by the neuroendocrine cells [25]. Exogenous incretin therapy has proven beneficial in patients with Type 2 $\mathrm{DM}$, but it has also been associated with weight loss in this cohort, an undesirable outcome for patients with CF [26]. Hyperglycaemia is known to promote beta-cell apoptosis, and as such, postprandial hyperglycaemia from dysfunctional incretin secretion in CF may potentiate the glycaemic abnormalities demonstrated and hasten the progression to CFRD.

CFRD is distinct from both Type 1 and Type 2 diabetes. CFRD is not an autoimmune condition like Type $1 \mathrm{DM}$ and is not associated with autoantibodies found in Type $1 \mathrm{DM}$. Moreover, Type $2 \mathrm{DM}$ is primarily a disorder of insulin resistance, whereas glucose abnormalities in CF are primarily the result of insulin deficiency, which is present even in CF patients with normal glucose tolerance on oral glucose tolerance test (OGTT) [7]. One of the features of CFRD that differentiates it from other forms of diabetes is the variation in glucose tolerance demonstrated over time [27]. Although abnormalities of glucose tolerance are known to progress and the complications of diabetes increase in the degree of abnormal glycaemia, some patients with the diagnosis of CFRD will have OGTT results that normalise [27]. The role of insulin resistance has been less well defined although there is emerging evidence of its importance. Ahmad et al. illustrated that patients with CF actually had an increase in peripheral insulin sensitivity compared to healthy controls matched for age and body mass index. They concluded that this increase in peripheral sensitivity in CF patients was a metabolic compensation for insulin deficiency [28]. Moran et al. replicated these findings in exocrine-insufficient CF patients without diabetes. However, once CFRD had developed, there was an increase in peripheral insulin resistance [29]. The mechanism by which this may occur could be the result of a downregulation of GLUT-4 insulin-sensitive channels secondary to chronic hyperglycaemia [30] ("glucose toxicity"). Insulin resistance is also thought to vary over time which could explain the variability of glucose tolerance seen in patients with $\mathrm{CF}$, including a normalisation of previously abnormal glucose tolerance on OGTT. It is often cited that glucose abnormalities worsen during pulmonary exacerbations (due 
to cytokine and stress hormone release), but the data to support this suggestion is limited and was not found in the study by Widger et al. [31]. This group performed OGTT in patients with a pulmonary exacerbation and then repeated the OGTT when well. Although the sample size was small, 8/9 patients remained within their glycaemic category even when recovered from their pulmonary exacerbation. However insulin resistance is known to increase during periods of corticosteroid usage, overnight feeds [32, 33], pregnancy and during puberty [34-36]. In the latter case, insulin resistance is thought to increase as a result of a physiological elevation in growth hormone [34], and this may account for the increased detection of CFRD in this age group.

Chronic inflammation may play a key role in the development of glucose abnormalities in CF. Bismuth et al. demonstrated in their cohort of patients with CF that the erythrocyte sedimentation rate (ESR), a marker of inflammation, positively correlated with $\mathrm{HbA}_{1 \mathrm{c}}$ and the area under the curve (AUC) for glucose in patients undergoing OGTT [37]. Significant and ongoing oxidative stress is one mechanism hypothesised to result in an inflammatory state and beta-cell apoptosis $[38,39]$. One review postulated that the imbalance in inflammatory T-cell lymphocytes known to play a role in the development of other forms of diabetes may contribute to lung inflammation and thereby a chronic inflammatory states resulting in glucose abnormalities [40]. T-helper 17 (Th-17) lymphocyte cells secrete a pro-inflammatory cytokine-IL-17 known to be involve in pulmonary inflammation in CF and is known to be present in higher levels compared to controls in patients with Type 2 diabetes. Furthermore, studies also suggest that IL-17 may play an important role in the development of Type 1 diabetes [41] and may contribute to $\beta$-cell destruction. It has also been postulated that cytokines such as TNF- $\alpha$ may act directly on the insulin receptor by inducing insulin resistance, thereby inhibiting the potential action of insulin [42].

The pathophysiology of CFRD is likely to be multifactorial but ultimately resulting from progressive insulin deficiency secondary to islet cell destruction and defective beta-cell secretion, combined with stressors that intermittently increase insulin resistance resulting in a further deterioration of glycaemic status. Certain patients may be more at risk if non-CFTR genetic modifiers are present $[13,14]$, and perhaps these patients are unable to compensate for the degree of histological pancreatic destruction and defective beta-cell functioning.

\section{Epidemiology}

\subsection{Prevalence of glycaemic abnormalities in CF}

CFRD is known to occur in up to $50 \%$ of patients with CF by the age of 30 years [43] and the prevalence increases with age. CFRD can occur in young children with CF but is rare [8]. Recent studies suggest that CFRD affects approximately 9\% in the 5-9 year age group [44] and a smaller proportion of children under 5 may also meet the CFRD diagnostic criteria. Yi et al. recently reported a series that suggested $5 \%$ of their cohort between 6 months and 5 years had CFRD [45]. Although a small proportion of young children have CFRD, the average age of onset is 20 years [46]. CFRD occurs more commonly in females with a prevalence of $17 \%$ in young female adults compared with $12 \%$ in males previously described [47]. 
Children with CF are known to be insulin deficient from birth. Milner et al. demonstrated that children with CF in the first year of life had lower insulin levels than controls [7]. Insulin deficiency will progress over time and results in a gradual deterioration of glucose tolerance. As such, impaired glucose tolerance is much more common than CFRD and can affect up to $41 \%$ of children in the $6-9$ year age group [48], compared with only $10 \%$ of this group being classified as CFRD. The risk of early CFRD is much higher in children with abnormal glucose tolerance on OGTT [48].

\subsection{Screening}

The prevalence of identified CFRD has been shown to increase after the introduction of screening [49]. Unlike Type 1 or Type 2 diabetes which are often symptomatic, CFRD does not often present with symptoms of hyperglycaemia although this can occur in approximately one third of patients. Symptoms can include polyuria and polydipsia, but CFRD is more likely to present insidiously with the catabolic complications of insulin deficiency such as nutritional deterioration or decline in pulmonary function. When routine screening was introduced in Australia, the incidence of CFRD increased from 2.0 to 22.1 per 1000 person years between 2000 and 2008, which represents a tenfold increase [50]. A decline in the age of diagnosis has also been demonstrated after the introduction of routine screening; Noronha et al. reported a reduction in the mean age of diagnosis from 22.3 years to 13.5 years [49]. Routine screening from at least 10 years of age with an OGTT is recommended by most guidelines [51, 52].

\subsection{Risk factors for CFRD}

The risk factors for the development of CFRD are closely linked to the specific CFTR genotype and the severity of the CFTR protein dysfunction [53]. CFTR mutations are classified according to the resulting functional deficit [54]. Class 1 and class 2 mutations result in the total or partial absence of CFTR protein at the surface membrane due to defective/non-functional protein (Class 1, e.g. stop codon mutations) or due to defective transfer of the protein to the cell membrane, i.e. defective "trafficking" (Class 2, e.g. F508) [25]. Classes 3, 4, 5 and 6 have irregularities in regulation, conductance, prevalence and stability of CFTR at the membrane [55]. Of the latter, 4 classes, all except class 3, which is known as a gating mutation, have partial function. Those classes with no action have a more severe phenotype and are associated with a greater risk of CFRD, such as homozygous F508 patients [46].

CFRD generally occurs in patients with pancreatic insufficiency. There have been reports of CFRD in patients who are pancreatic sufficient, but the diagnostic criteria for exocrine pancreatic function do not appear to be robust [47]. Some of these patients were classified as pancreatic sufficient because they were not taking replacement enzymes, but had not undergone any formal diagnostic testing such as faecal elastase or 3-day fat stool sampling. More recent studies have demonstrated that the degree of pancreatic exocrine function appears to correlate with the development of CFRD. Soave et al. demonstrated a causal relationship between the level of serum trypsinogen on the newborn screen (a marker of exocrine pancreatic function used to diagnose CF) and the development of CFRD over time [15]. Trypsinogen is an inactive pancreatic enzyme precursor required for protein digestion and absorption. It is 
converted to trypsin when secreted into the small intestine, but this process is inhibited in CF and results in an elevated serum trypsinogen. A significant elevation in the blood levels of immunoreactive trypsinogen (IRT) on newborn screening is used to identify neonates with $\mathrm{CF}$. The IRT level is known to decline rapidly over $\mathrm{T}$ ime with ongoing pancreatic destruction. Soave et al. postulated that patients with CF who had more significant pancreatic disease at birth would have IRT levels that had already started to decline and would be relatively lower than the rest of the CF cohort [15]. They also demonstrated that those children with relatively low IRT amongst the CF cohort had an increased risk of CFRD, thus confirming the relationship between exocrine pancreatic function and endocrine disease.

The presence of CF liver disease appears to be a significant risk factor in the development of CFRD. Leung et al. examined over 700 liver ultrasounds of patients with CF and found that patients with the features of heterogenous or cirrhotic liver disease on ultrasound were more likely to have abnormalities of glucose tolerance, including CFRD, than those with normal liver ultrasounds [56]. The relationship between liver disease and CFRD remains unclear. It could be a result of the more severe genotypes causing CFRD also increasing the risk of liver disease, or it could be the result of a non-CFTR genetic modifier.

Abnormal glucose tolerance is a known risk factor for progression to CFRD. CF patients with glucose abnormalities are up to 11 times more likely to develop early CFRD than other 6-9-year-old patients [48].

\section{The clinical impact of glucose abnormalities in CF}

Glucose abnormalities in CF are associated with significantly increased morbidity and mortality [2]. Prior to the introduction of routine screening for CFRD, less than $25 \%$ of CFRD patients survived to age 30 , compared with $60 \%$ of patients without diabetes [57]. When Moran et al. examined female CF cohorts with and without CFRD in the 1990s and compared them with cohorts after the introduction of routine CFRD screening, mortality rates had halved: 6.9 per 100 patients years in patients with CFRD versus 3.2 per 100 patient years in CF without diabetes, with similar results seen in men were reported [58]. Although mortality rates for patients with CFRD have seen a marked improvement, a significant difference between CF patients with and without diabetes persists [59].

CFRD leads to a significant increase in respiratory exacerbations, increased infection with CF pathogens [60] (including Pseudomonas aeruginosa) and poorer lung function [57, 61]. The mechanism by which insulin deficiency resulting in CFRD has such a negative impact on lung function in CF is probably multifactorial. The hyperglycaemic environment is said to create a "pro-inflammatory" environment, optimal for bacterial growth and colonisation that allows CF pathogens to thrive [52]. In vitro studies have demonstrated an amplification of bacterial growth, in particular Staphylococcus aureus and P. aeruginosa with increasing glucose concentrations [62], and this evidence supports the hypothesis of glycaemic abnormalities playing a significant and direct role in the infection and colonisation of patients with CF. 
Blood glucose levels $>8 \mathrm{mmol} / \mathrm{L}$ correlate with increased airway glucose levels in patients with CF [62]. In non-CF patients, elevated airway glucose has been demonstrated to be a risk factor for respiratory infections, including MRSA (based on studies in patients intubated due to critical illness in the intensive care unit [63]). When Brennan et al. examined the airway glucose of patients with $\mathrm{CF}$, they demonstrated that even patients with normal glucose tolerance on OGTT had glucose in their airway for longer periods of time than the control population. The duration of time spent with airway glucose levels $>8 \mathrm{mmol} / \mathrm{L}$ correlated with the degree of glucose abnormality [62]. The level at which glucose appears in the airway is much lower than the 2-h OGTT glycaemic threshold for CFRD and also appears to be very close to the level of blood glucose level (BGL) which correlates with significant nutritional and respiratory decline [64].

Respiratory tract infections may not entirely account for the deterioration in lung function seen in patients with CF. Patients with diabetes mellitus from other causes have also been demonstrated to have poorer lung function than matched controls, even in the absence of respiratory disease $[65,66]$. It is unclear whether this is a direct result of glucose in the airways or an indirect result of inflammation from relative insulin deficiency.

Nutrition in CF has a direct correlation with survival [67], and insulin, an anabolic hormone, plays an integral role in maintaining weight and building muscle [18]. When CF patients are insulin deficient, this manifests as poorer nutritional status. Multiple studies have demonstrated the impact of CFRD and insulin deficiency on nutrition and growth [37]. The data of over 8000 CF patients on the epidemiologic study of cystic fibrosis (ESCF) was analysed in 2005 and confirmed a greater impairment in nutrition in the CFRD group when compared with the nondiabetic group [47]. The CFRD cohort had statistically lower height for age percentiles, weight for age percentiles and BMI $(\mathrm{p}<0.001$ for all three parameters). A statistically significant difference in body weight and BMI has also been demonstrated in the "prediabetic" CF patients when compared with CF patients with normal glucose tolerance [61]. This decline was detected by Lanng et al. in some patients 4 years prior to the diagnosis of CFRD being. Given the insidious nature of glycaemic abnormalities and the inherent difficulties with nutrition in patients with $\mathrm{CF}$, particularly those with exocrine pancreatic insufficiency, the impact of insulin deficiency is often not recognised until CFRD is diagnosed on routine screening.

\section{The spectrum of glucose abnormalities in CF}

Insulin deficiency is progressive and results in a deterioration of glucose tolerance over time. CFRD lies at the end of a spectrum of glucose abnormalities. Glycaemic categories in CF are determined based on the results of the oral glucose tolerance test (OGTT) [51]. To perform an OGTT, a glucose load of $1.75 \mathrm{~g} / \mathrm{kg}$ (maximum $75 \mathrm{~g}$ ) is consumed after fasting. Classically the blood glucose level (BGL) is measured at 0 and $120 \mathrm{~min}$ [68]. Additional information about glucose tolerance is gained by also checking the BGL at $30 \mathrm{~min}, 60 \mathrm{~min}$ and $90 \mathrm{~min}$, i.e. a 30-min sampled OGTT [64].

The diagnosis of CFRD is made based on the American Diabetic Association (ADA) criteria [51] (see Table 1). CFRD is diagnosed when the 2-h OGTT level is $\geq 11.1 \mathrm{mmol} / \mathrm{L}$ and can 


\begin{tabular}{llll}
\hline Category & Fasting level & Midpoint peak (1 h) & 2-h plasma level \\
\hline Normal glucose tolerance & $<7 \mathrm{mmol} / \mathrm{L}$ & $<11.1 \mathrm{mmol} / \mathrm{L}$ & $<7.8 \mathrm{mmol} / \mathrm{L}$ \\
$\begin{array}{l}\text { Indeterminate glycaemia } \\
\text { (INDET) }\end{array}$ & $<7 \mathrm{mmol} / \mathrm{L}$ & $\geq 11.1 \mathrm{mmol} / \mathrm{L}$ & $<7.8 \mathrm{mmol} / \mathrm{L}$ \\
$\begin{array}{l}\text { Impaired glucose tolerance } \\
\text { (IGT) }\end{array}$ & $<7 \mathrm{mmol} / \mathrm{L}$ & \\
$\begin{array}{l}\text { CFRD without fasting } \\
\text { hyperglycaemia }\end{array}$ & $<7 \mathrm{mmol} / \mathrm{L}$ & & \\
$\begin{array}{l}\text { CFRD with fasting } \\
\text { hyperglycaemia }\end{array}$ & $\geq 7 \mathrm{mmol} / \mathrm{L}$ & & \\
\hline
\end{tabular}

Table 1. Classification of abnormalities of glucose tolerance in cystic fibrosis on OGTT.

occur with or without fasting hyperglycaemia (fasting BGL $\geq 7.0 \mathrm{mmol} / \mathrm{L}$ is defined as fasting hyperglycaemia). Fasting hyperglycaemia can also be considered diagnostic of CFRD, if still abnormal when repeated. One fasting BGL $\geq 7.0 \mathrm{mmol} / \mathrm{L}$ and another non-fasting level $\geq 11.1 \mathrm{mmol} / \mathrm{L}$ can also make a diagnosis of CFRD. If a patient is sick and glycaemic abnormalities persist for two days, then the diagnosis can also be made. Most guidelines recommend the OGTT/BGL is repeated before the diagnosis is confirmed. Some guidelines subclassify CFRD based on the fasting BGL, but this distinction does not alter management, as insulin treatment is recommended for those with and without fasting hyperglycaemia.

Additional criteria have been published to subclassify the patients into glycaemic categories based on 30-min samples (see Table 1) [1]. Patients with normal glucose tolerance have fasting BGL $<7.0 \mathrm{mmol} / \mathrm{L}$ and 2-h level $<7.8 \mathrm{mmol} / \mathrm{L}$. Indeterminate glycaemia (INDET) is defined as normal fasting and 2-h levels with a midpoint level $\geq 11.1 \mathrm{mmol} / \mathrm{L}$. Impaired glucose tolerance (IGT) is defined by a 2-h level $<11.1 \mathrm{mmol} / \mathrm{L}$ but $\geq 7.8 \mathrm{mmol} / \mathrm{L}$.

Children with abnormal glucose tolerance and CF may fluctuate between glycaemic categories because of increasing insulin requirements at times of illness or because of variable levels of resistance. In one study $18 \%$ of CF patients with abnormal glucose tolerance had glycaemic abnormalities that improved over time. Twenty-two percent of patients had a deterioration in their glucose tolerance [27]. This variability was replicated by Lanng et al. who saw a normalisation of the patient OGTT in 58\% of adult patients with CF when followed up after 5 years [69]. Yi et al. examined glucose tolerance in young children ( $<6$ years) and found that some of these children with abnormal glucose tolerance that normalised, including those that met CFRD criteria [45]. This variability adds to the difficulty seen in managing patients with CFRD, particularly younger children.

\section{Issues with the OGTT in CFRD}

The OGTT was not designed to diagnose diabetes in the CF population. The test was designed to determine the treatment threshold for Pima Native American population with Type 2 diabetes based on their risk of developing microvascular complications [70]. 
Although microvascular diabetes complications can occur in $\mathrm{CF}$, the major concern for CFRD is its impact on nutrition and lung function. Complications from chronic intermediate hyperglycaemia may also result in microvascular disease prior to the patient meeting the criteria for CFRD [71]. More practical goals would include an initiation of treatment at a time that would alleviate significant respiratory morbidity such as recurrent infections and respiratory function decline. The drop in nutritional status and weight, or poor growth in younger children because of insulin deficiency catabolism, would be a more relevant CF-specific outcome to guide diagnostic targets.

The decrease in lung function and nutrition seen in CFRD actually precedes the diagnosis by several years and is often insidious. Lanng et al. noted that a decline was present up to four years prior to the OGTT 2-h criteria being met [61]. Furthermore, insulin therapy has been demonstrated to reverse some of the nutritional decline seen in patients with abnormal glycaemia $[72,73]$. However, once patients meet the criteria for CFRD, recovery of lung function is not always possible. Widger et al. postulate that by waiting until the patient meets the CFRD criteria to start insulin, the conceded progression from abnormal glucose tolerance to CFRD allows irreversible structural remodelling of the lungs that cannot be corrected with insulin therapy [74].

Further evidence for insulin therapy at an earlier stage of the glycaemic spectrum is warranted, and initial data has highlighted which patients may benefit most. Schmid et al. demonstrated that in 1000 patients with CF, patients with midpoint level $\geq 11.1 \mathrm{mmol} / \mathrm{L}$ (INDET) were predictive for later development of CFRD [75]. Brodsky et al. were able to establish that the 1-h level on the OGTT correlated with poorer lung function [76]. They examined 101 patients with CF and these patients with higher 1-h levels had poorer respiratory status even when corrected for nutritional status. The 2-h "diagnostic" level in this group did not correlate with BMI or lung function. The findings of Coriati et al. [77] confirm that waiting for the 2-h BGL to be diagnostic of CFRD may be too late. Their cohort of patients with indeterminate glycaemia already had significant loss of lung function, equivalent to the lung function of patients with newly diagnosed CFRD. The criteria to start insulin in the future may be determined by the patient's own risk of developing CFRD or by early clinical signals in lung function and intermediate glucose abnormalities.

Hameed et al. used a 30-min sampled OGTT and found that a peak BGL $\geq 8.2 \mathrm{mmol} / \mathrm{L}$ was reliably predictive of a decline in lung function and nutrition in the preceding year [64]. Based

\begin{tabular}{lll}
\hline Proposed new criteria & Peak blood glucose $\left(\mathrm{BG}_{\max }\right) \mathbf{m m o l} / \mathrm{L}$ & Blood glucose at $\mathbf{1 2 0 ~} \mathbf{m i n} \mathbf{~ m m o l} / \mathbf{L}$ \\
\hline CFID1 & $\geq 8.2$ & $<11.1$ \\
CFID2 & $\geq 11.1$ & $<11.1$ \\
CFID3 & & $\geq 11.1$ \\
& & Without fasting hyperglycaemia \\
CFID4 & $\geq 11.1$ \\
& & With fasting hyperglycaemia \\
\hline
\end{tabular}

CFID = cystic fibrosis insulin deficiency.

Table 2. Proposed new staging criteria for insulin deficiency and early glucose abnormalities in CF, based on the OGTT with samples every $30 \mathrm{~min}$. 
on these results, this group proposed a new staging criteria to identify insulin deficiency and early glucose abnormalities in patients with CF (see Table 2) [78]. Cystic fibrosis insulin deficiencies (CFID) 4 and 3 correspond to existing CFRD categories with and without fasting hyperglycaemia, respectively. CFID 1 and 2 are earlier stages of insulin deficiency that are distinct from impaired glucose tolerance (IGT) because they are based on the peak glucose level and have 2 -h levels $<11.1$. CFID 1 is defined by a midpoint peak glucose level $\geq 8.2 \mathrm{mmol} / \mathrm{L}$, and CFID2 has a midpoint glucose peak $\geq 11.1 \mathrm{mmol} / \mathrm{L}$.

\section{Continuous glucose monitoring in $\mathrm{CF}$}

Continuous glucose monitoring (CGM) has been used for several years in the management of Type 1 diabetes although it is not licenced for use as a diagnostic device. CGM uses a small probe inserted into the subcutaneous space where it measures interstitial glucose levels. Inserting the device is a relatively simple procedure that can be done within a few minutes in a clinic environment. It is easy to remove at home by the patient or carer, without any specific medical training. The device averages the glucose readings every five minutes and can be worn for several days whilst the patient continues to participate in normal activities and consumes their normal diet. The CGM device has been validated in CF and non-CF populations and shown to correlate with plasma glucose measurements $[79,80]$. When compared with OGTT, CGM appears to be reproducible and a reliable assessment of glycaemic abnormalities. When used in Type 1 diabetes, Bergenstal was able to demonstrate that children and adults on insulin pumps had improved glycaemic control, as measured by $\mathrm{HbA}_{1 \mathrm{c}}$ than those who did not use CGM [81].

CGM may be particularly useful in managing cystic fibrosis. CF patients frequently demonstrate early postprandial hyperglycaemia [79, 82, 83], reflected by elevations in readings on a 30-min sampled OGTT in the setting of a normal 2-h level. This intermittent postprandial hyperglycaemia may be reflected in the poor correlation of $\mathrm{HbA}_{1 \mathrm{c}}$ (glycated haemoglobin) with early glycaemic abnormalities in $\mathrm{CF}$. $\mathrm{HbA}_{1 \mathrm{c}}$ represents an index of the average of blood glucose concentrations in the preceding 2-3-month period, and the result is influenced by the half-life of the red cells [84]. When measured in CF, it is a poor indicator of glycaemic abnormalities as it is often still normal by the time a diagnosis of CFRD has been made. The poor sensitivity of the test may result from the intermittent nature of hyperglycaemia in patients with $\mathrm{CF}$, which is not revealed in the $\mathrm{HbA}_{1 \mathrm{c}}$ level when the glucose levels are "averaged", as well as increased red cell turnover in $\mathrm{CF}$.

CGM provides a useful tool to guide insulin treatment once the diagnosis of CFRD has been made [79], but it may also offer a potential opportunity to capture the moments of postprandial hyperglycaemia in CF in the screening and diagnostic phase. In CF patients with normal glucose tolerance on OGTT, abnormalities on CGM have been detected [79, 82, 83]. This could reflect the fact that patients with CF undergo a period of fasting prior to their glucose load in the OGTT which will only measure two values. When a CGM is worn, patients can be at home and may consume their normal CF diet including a carbohydrate load that may exceed the glucose level consumed during an OGTT. In the same way that $\mathrm{HbA}_{1 \mathrm{c}}$ may not reflect a true 
picture of glycaemic abnormalities in CF, so too may the OGTT underestimate the hyperglycaemia in these patients, particularly in the early phase of glucose abnormalities.

CGM may be a useful device in predicting which children with CF will develop glycaemic abnormalities. Schiaffini et al. performed OGTT and CGM on children with CF and then repeated the OGTT after 2 years. Children who had diabetic excursions on CGM at baseline, even those with normal glucose tolerance on OGTT, developed impaired glucose tolerance or CFRD when the OGTT was repeated 2 years later [83]. Initial data on CGM does appear to suggest that this tool may be useful in identifying clinically significant glucose abnormalities in CF. Leclercq et al. demonstrated, in a CF population with normal OGTT, that patients who recorded glucose levels in the diabetic range $(\geq 11.1 \mathrm{mmol} / \mathrm{L})$ on CGM had poorer lung function and greater colonisation with CF respiratory pathogens such as $P$. aeruginosa [85].

Glycaemic abnormalities are known to have a significant impact on nutrition in patients with CF. CGM may provide an opportunity to highlight which children are at risk of nutritional decline secondary to abnormities of glucose tolerance as described in the study by Hameed et al. [64]. In this study of 25 children with CF undergoing CGM, if $\geq 4.5 \%$ of the study duration was spent with an interstitial glucose reading $>7.8 \mathrm{mmol} / \mathrm{L}$, this was predictive of a decline in weight standard deviation score. This CGM criterion had a sensitivity of $89 \%$ and a specificity of $86 \%$ in detecting this nutritional decline. CGM abnormalities do appear to be clinically significant, but there are not studies as yet demonstrating a benefit from treatment based on CGM recordings in $\mathrm{CF}$, and the device is not yet licenced to make a diagnosis of diabetes.

\section{Management of CFRD}

The main aim of CFRD treatment is to correct the hyperglycaemia and its downstream effects on respiratory function and infections, in addition to reversing significant protein catabolism secondary to insulin deficiency. Optimal management has been shown to improve lung function and morbidity [72]. Although a drop in mortality from late CFRD diagnosis has been seen, the risk of early mortality is still higher in this population. The mainstay of treatment is exogenous insulin therapy, but studies are underway examining the benefits of dietary changes and the use of oral hypoglycaemic agents in CF.

\subsection{Insulin}

Insulin plays a major role in the management of CFRD. Insulin replacement by subcutaneous injection in CFRD has been shown to improve lung function and reduce pulmonary exacerbation frequency [86]. It has also been shown to benefit the nutritional status of the patient, with an improvement in growth seen in children with CF [73]. Recent studies have also demonstrated that insulin therapy in the prediabetic phase may also play a valuable role in the management of patients with CF. Hameed et al. were able to replicate previous studies demonstrating a benefit of insulin therapy on lung function and nutrition in patients with $\mathrm{CF}$ and revealed an improvement in weight standard deviation score $(p=0.003)$ and lung function (FEV1 improvement $\mathrm{p}=0.004$ ) with once daily insulin injections (detemir, Levemir ${ }^{\mathrm{TM}}$ ) [73]. 
Insulin is given via subcutaneous injection. Unlike Type 1 diabetes, a once daily dose of long-acting insulin may be all that is required to demonstrate a benefit for this population [73]. Insulin doses vary with each patient, but because of the important anabolic role insulin plays in growth and nutrition, the highest tolerated dose without hypoglycaemia or other side effects is generally recommended [52] (taking into account patient-specific factors such as ability to recognise hypoglycaemic symptoms). The dose prescribed may vary over time with increasing requirements during times of relative increase in insulin resistance such as with glucocorticoid use or during periods of growth and pregnancy. Given the progressive nature of insulin deficiency in $\mathrm{CF}$, increasing requirements may be seen over time, particularly in the paediatric population with CF that have age- and weight-based doses.

Insulin pumps that continuously deliver a small amount of insulin into the subcutaneous space have been used in patients with CFRD [87] although the uptake in CF has been poor when compared with other forms of diabetes. When wearing a pump, patients are currently required to undertake much more intensive finger-prick blood glucose testing than that required with a once daily insulin injection. This may prove to be too onerous for patients with CFRD who already have a significant treatment burden with multiple oral and nebulised medications and physiotherapy. Future insulin pump devices may include closed loop systems, in which interstitial glucose levels measured by CGM calibrate the rate and amount of insulin secreted by the pump [88]. These devices are currently under investigation for Type $1 \mathrm{DM}$, but there are no data published about their use in CFRD to date.

\subsection{Nutrition}

Nutritional education and support are of utmost importance for patients with a diagnosis of CFRD. Children with CF require a higher caloric intake (may need up to $200 \%$ of usual recommendations [89]) to achieve optimal nutritional and growth targets. If nutritional targets are not met, there may be significant consequences as a lower BMI has been associated with increased mortality in CF [67]. These additional calories are best taken from fat and protein-based meals, but a significant proportion is taken from carbohydrates [90]. Patients with abnormalities of glucose tolerance and CFRD will be required to recognise carbohydrates in their diet, as the carbohydrate load will affect the glucose level and the resulting insulin requirements. This is usually done by educating the family and patient about carbohydrate-insulin ratios.

There are very limited data regarding the dietary management of CFRD. This is of particular significance given that hyperglycaemia has been demonstrated to worsen glycaemic abnormalities in CF, possibly by potentiating beta-cell apoptosis. As such, glycaemic control in CFRD needs to be tight, and diets that perpetuate postprandial hyperglycaemia may have a negative impact on glycaemic abnormalities in CF and increase insulin requirements. A low glycaemic diet is often recommended in Type 1 and Type 2 diabetes to optimise control of hyperglycaemia and has been shown to decrease insulin requirements and improve glucose homeostasis, without having a significant impact on quality of life for these patients. Whereas weight loss due to change in diet may be beneficial in Type $2 \mathrm{DM}$, this may have serious negative consequence in CF. There is not enough information in the literature to recommend any dietary changes that might improve glycaemic control or prevent or delay progression to CFRD if instituted at an earlier stage. 


\subsection{Oral hypoglycaemic agents}

Oral agents do not play a role in the management of patients with CFRD. Many agents target insulin resistance (e.g. metformin), which is not a major feature in the early glycaemic abnormalities of CF where insulin deficiency plays the key role and as such will not be of significant benefit to CF patients. Significant side effects from oral hypoglycaemic agents such as hepatotoxicity are a serious complication for the CF population where a significant proportion may develop CF liver disease [91]. Insulin therapy in states of insulin deficiency such as Type 1 diabetes has been shown to preserve insulin secretion and "rest" the residual beta cells. Conversely, agents that stimulate insulin secretion may potentially hasten beta-cell loss. For example, agents such as repaglinide may be useful in the short term but ultimately have a negative long-term impact.

\subsection{The role of potentiators in CFRD}

Evidence for the use of potentiators in CFRD is limited, but a few pilot studies have been published that suggest a benefit on glucose homeostasis in CF. In a single pair of CF siblings with abnormal glucose tolerance (one with CFRD) and gating mutations, a reduction in the glucose AUC and an improvement in the insulin secretion profile was demonstrated after the introduction of ivacaftor $\left(\right.$ Kalydeco $\left.^{\mathrm{TM}}\right)$. Bellin et al. also demonstrated improvements in glucose homeostasis after the introduction of ivacaftor. In this group of five CF patients with glucose abnormalities, four of five demonstrated improvements in insulin secretion. The patient whose insulin secretion did not improve had long-standing CFRD, whereas the others had earlier glycaemic abnormalities. Theoretically, the patient with long-standing CFRD could already have undergone such significant pancreatic destruction that the abnormalities of glucose tolerance could not be corrected at the level of the CFTR.

\section{Complications of CFRD}

Long-standing hyperglycaemia and insulin deficiency will result in an increase in respiratory exacerbations and morbidity and poorer nutrition. It will also result in complications from chronic hyperglycaemia seen in other forms of diabetes. Historically the life-limiting nature of $\mathrm{CF}$ and in particularly those with CFRD meant that CF patients were unlikely to live long enough to develop end-organ dysfunction from the macrovascular and microvascular complications seen in other forms of diabetes. With an improvement in life expectancy, these long-term issues need to be addressed, and routine screening needs to be a part of CF clinical care. This will include examination for neuropathy and retinopathy and urine screening for microalbuminuria. In one study, 10 years after the diagnosis of CFRD has been made, subjects with fasting glycaemia demonstrated rates of microalbuminuria of approximately $14 \%$, retinopathy $16 \%$, neuropathy 55\% and autonomic gastropathy 50\% [51]. Gilchrist et al. reported retinopathy in three patients with abnormal glucose tolerance but not meeting criteria for CFRD [71] which further supports the proposition that the OGTT may not be the ideal test for significant glycaemic abnormalities in patients with CF. 


\section{Conclusion}

Cystic fibrosis-related diabetes continues to pose a significant risk of increased morbidity and mortality to the CF population. However, CFRD lies at the endpoint of spectrum of glucose abnormalities, and increasing evidence implies that earlier glycaemic abnormalities may also be clinically significant. The standard OGTT does not appear to be sensitive enough to pick up early, clinically significant abnormalities of glucose tolerance secondary to insulin deficiency and the dysregulation of insulin secretion detected in CF patients. Hyperglycaemia in CF affects lung function, risk of respiratory pathogens, nutrition and growth in young children, and treating teams need to be proactive in the screening and diagnosis of glycaemic abnormalities that may be insidious and potentially irreversible if recognised late. Early recognition of hyperglycaemia in CF is required to prevent significant morbidity. Novel techniques such as continuous glucose monitoring may play a role in screening and early identification of at risk patients, as they have been shown to be predictive of significant glucose abnormalities in the future such as CFRD, but there is not enough evidence as yet to recommend their routine use in diagnosis. Future directions may include the use of potentiators and correctors in CF which appear to have potential to correct abnormalities of glucose tolerance but may be limited if instituted late and once significant pancreatic destruction has occurred.

\section{Acknowledgements}

SH and CFV are grateful for funding assistance from the National Health and Medical Research Council of Australia, Australasian Cystic Fibrosis Research Trust, Regional Diabetes Support Scheme, Sydney Children's Hospital Foundation and Australasian Paediatric Endocrine Care Grant from Pfizer and for industry support from Novo Nordisk, Medtronic and Abbott Diagnostics. BP has been awarded a scholarship from the Thoracic Society of Australia and New Zealand and Vertex.

\section{Author details}

Bernadette Prentice ${ }^{1,2^{*}}$, Shihab Hameed ${ }^{2,3}$, Chee Y. Ooi ${ }^{2,4}$, Charles F. Verge ${ }^{2,3}$ and John Widger ${ }^{1,2}$

*Address all correspondence to: bernadette.prentice@health.nsw.gov.au

1 Department of Respiratory Medicine, Sydney Children's Hospital, Randwick, NSW, Australia

2 Discipline of Paediatrics, School of Women's and Children's Health, The University of New South Wales, Sydney, NSW, Australia

3 Department of Endocrinology, Sydney Children's Hospital, Randwick, NSW, Australia

4 Department of Gastroenterology, Sydney Children's Hospital, Randwick, NSW, Australia 


\section{References}

[1] Hameed, S., Jaffe, A., and Verge, C.F., Advances in the detection and management of cystic fibrosis related diabetes. Curr Opin Pediatr, 2015. 27(4): pp. 525-33.

[2] Chamnan, P., et al., Diabetes as a determinant of mortality in cystic fibrosis. Diabetes Care, 2010. 33(2): pp. 311-6.

[3] Couce, M., et al., Diabetes mellitus in cystic fibrosis is characterized by islet amyloidosis. J Clin Endocrinol Metab, 1996. 81(3): pp. 1267-72.

[4] Iannucci, A., et al., Endocrine pancreas in cystic fibrosis: an immunohistochemical study. Hum Pathol, 1984. 15(3): pp. 278-84.

[5] Sequeiros, I., et al., MRI appearance of the pancreas in patients with cystic fibrosis: a comparison of pancreas volume in diabetic and non-diabetic patients. British J Radiol, 2014.

[6] Abdul-Karim, F.W., et al., Islets of Langerhans in adolescents and adults with cystic fibrosis. A quantitative study. Arch Pathol Lab Med, 1986. 110(7): pp. 602-6.

[7] Milner, A.D., Blood glucose and serum insulin levels in children with cystic fibrosis. Arch Dis Child, 1969. 44(235): pp. 351-5.

[8] Gelfand, I.M., Eugster, E.A., and Haddad, N.G., Infancy-onset cystic fibrosis-related diabetes. Diabetes Care, 2005. 28(10): pp. 2593-4.

[9] Olivier, A.K., et al., Abnormal endocrine pancreas function at birth in cystic fibrosis ferrets. J Clin Invest, 2012. 122(10): pp. 3755-68.

[10] Uc, A., et al., Glycaemic regulation and insulin secretion are abnormal in cystic fibrosis pigs despite sparing of islet cell mass. Clin Sci (Lond), 2015. 128(2): pp. 131-42.

[11] Bellin, M.D., et al., Insulin secretion improves in cystic fibrosis following ivacaftor correction of CFTR: a small pilot study. Pediatr Diabetes, 2013. 14(6): pp. 417-21.

[12] Tsabari, R., et al., CFTR potentiator therapy ameliorates impaired insulin secretion in CF patients with a gating mutation. J Cyst Fibros, 2015.

[13] Blackman, S.M., et al., A susceptibility gene for type 2 diabetes confers substantial risk for diabetes complicating cystic fibrosis. Diabetologia, 2009. 52(9): pp. 1858-65.

[14] Blackman, S.M., et al., Genetic modifiers play a substantial role in diabetes complicating cystic fibrosis. J Clin Endocrinol Metab, 2009. 94(4): pp. 1302-9.

[15] Soave, D., et al., Evidence for a causal relationship between early exocrine pancreatic disease and cystic fibrosis-related diabetes: a Mendelian randomization study. Diabetes, 2014. 63(6): pp. 2114-9.

[16] Gold, G., Gishizky, M.L., and Grodsky, G.M., Evidence that glucose "marks" beta cells resulting in preferential release of newly synthesized insulin. Science, 1982. 218(4567): pp. 56-8. 
[17] Daniel, S., et al., Identification of the docked granule pool responsible for the first phase of glucose-stimulated insulin secretion. Diabetes, 1999. 48(9): pp. 1686-90.

[18] Newsholme, P., et al., Nutrient regulation of insulin secretion and action. J Endocrinol, 2014. 221(3): pp. R105-20.

[19] Del Prato, S., Marchetti, P., and Bonadonna, R.C., Phasic insulin release and metabolic regulation in type 2 diabetes. Diabetes, 2002. 51(1): pp. S109-16.

[20] Jing, X., et al., Ca V 2.3 calcium channels control second-phase insulin release. J Clin Invest, 2005. 115(1): pp. 146-54.

[21] Kelly, A., and Moran, A., Update on cystic fibrosis-related diabetes. J Cyst Fibros, 2013. 12(4): pp. 318-31.

[22] Caumo, A., and Luzi, L., First-phase insulin secretion: does it exist in real life? Considerations on shape and function. Am J Physiol Endocrinol Metab, 2004. 287(3): pp. E371-85.

[23] O'Donovan, D., et al., Effects of lipase inhibition on gastric emptying of, and on the glycaemic, insulin and cardiovascular responses to, a high-fat/carbohydrate meal in type 2 diabetes. Diabetologia, 2004. 47(12): pp. 2208-14.

[24] Perano, S., et al., Cystic fibrosis related diabetes--a new perspective on the optimal management of postprandial glycemia. J Diabetes Complications, 2014. 28(6): pp. 904-11.

[25] Barrio, R., Management of endocrine disease: cystic fibrosis-related diabetes: novel pathogenic insights opening new therapeutic avenues. Eur J Endocrinol, 2015. 172(4): pp. R131-41.

[26] Amori, R.E., Lau, J., and Pittas, A.G., Efficacy and safety of incretin therapy in type 2 diabetes: systematic review and meta-analysis. JAMA, 2007. 298(2): pp. 194-206.

[27] Milla, C.E., Warwick, W.J., and Moran, A., Trends in pulmonary function in patients with cystic fibrosis correlate with the degree of glucose intolerance at baseline. Am J Respir Crit Care Med, 2000. 162(3 Pt 1): pp. 891-5.

[28] Ahmad, T., Nelson, R., and Taylor, R., Insulin sensitivity and metabolic clearance rate of insulin in cystic fibrosis. Metabolism, 1994. 43(2): pp. 163-7.

[29] Moran, A., et al., Insulin sensitivity in cystic fibrosis. Diabetes, 1994. 43(8): pp. 1020-6.

[30] Hardin, D.S., et al., Mechanisms of insulin resistance in cystic fibrosis. Am J Physiol Endocrinol Metab, 2001. 281(5): pp. E1022-8.

[31] Widger, J., et al., Glucose tolerance during pulmonary exacerbations in children with cystic fibrosis. PLoS One, 2012. 7(9): pp. e44844.

[32] MacDonald, A., Nutritional management of cystic fibrosis. Arch Dis Child, 1996. 74(1): pp. 81-7.

[33] Smith, D., Clarke, J., and Stableforth, D., A nocturnal nasogastric feeding programme in cystic fibrosis adults. J Hum Nutr Diet, 1994. 7(4): pp. 257-62. 
[34] Amiel, S.A., et al., Impaired insulin action in puberty. A contributing factor to poor glycemic control in adolescents with diabetes. N Engl J Med, 1986. 315(4): pp. 215-9.

[35] Hardin, D.S., et al., The metabolic effects of pregnancy in cystic fibrosis. Obstet Gynecol, 2005. 106(2): pp. 367-75.

[36] Moran, A., Endocrine complications of cystic fibrosis. Adolesc Med Clin, 2002. 13(1): p. 145.

[37] Bismuth, E., et al., Glucose tolerance and insulin secretion, morbidity, and death in patients with cystic fibrosis. The Journal of Pediatrics, 2008. 152(4): pp. 540-5.e1.

[38] Ntimbane, T., et al., Cystic fibrosis-related diabetes: from CFTR dysfunction to oxidative stress. Clin Biochem Rev, 2009. 30(4): pp. 153-77.

[39] Galli, F., et al., Oxidative stress and antioxidant therapy in cystic fibrosis. Biochim Biophys Acta, 2012. 1822(5): pp. 690-713.

[40] Ziai, S., et al., Could T cells be involved in lung deterioration and hyperglycemia in cystic fibrosis? Diabetes Res Clin Pract, 2014. 105(1): pp. 22-9.

[41] Roep, B.O., and Peakman, M., Diabetogenic T lymphocytes in human type 1 diabetes. Curr Opin Immunol, 2011. 23(6): pp. 746-53.

[42] Hotamisligil, G.S., et al., IRS-1-mediated inhibition of insulin receptor tyrosine kinase activity in TNF-alpha- and obesity-induced insulin resistance. Science, 1996. 271(5249): pp. 665-8.

[43] Lanng, S., et al., Diabetes mellitus in Danish cystic fibrosis patients: prevalence and late diabetic complications. Acta Paediatr, 1994. 83(1): pp. 72-7.

[44] Moran, A., et al., Abnormal glucose metabolism in cystic fibrosis. J Pediatr, 1998. 133(1): pp. 10-7.

[45] Yi, Y., et al., Abnormal glucose tolerance in infants and young children with cystic fibrosis. American Journal of Respiratory and Critical Care Medicine, 2016.

[46] Rosenecker, J., et al., Genetic determination of diabetes mellitus in patients with cystic fibrosis. Multicenter Cystic Fibrosis Study Group. J Pediatr, 1995. 127(3): pp. 441-3.

[47] Marshall, B.C., et al., Epidemiology of cystic fibrosis-related diabetes. J Pediatr, 2005. 146(5): pp. 681-7.

[48] Ode, K.L., et al., Oral glucose tolerance testing in children with cystic fibrosis. Pediatr Diabetes, 2010. 11(7): pp. 487-92.

[49] Noronha, R.M., et al., Importance of screening with oral glucose tolerance test for early diagnosis of cystic fibrosis-related diabetes mellitus. Pediatr Diabetes, 2014. 15(4): pp. 309-12.

[50] Rana, M., et al., Increased detection of cystic-fibrosis-related diabetes in Australia. Arch Dis Child, 2011. 96(9): pp. 823-6.

[51] Moran, A., et al., Clinical care guidelines for cystic fibrosis-related diabetes: a position statement of the American Diabetes Association and a clinical practice guideline of the Cystic Fibrosis 
Foundation, endorsed by the Pediatric Endocrine Society. Diabetes Care, 2010. 33(12): pp. 2697-708.

[52] Moran, A., et al., ISPAD Clinical Practice Consensus Guidelines 2014. Management of cystic fibrosis-related diabetes in children and adolescents. Pediatric Diabetes, 2014.15 (20): pp. 65-76.

[53] Adler, A.I., et al., Genetic determinants and epidemiology of cystic fibrosis-related diabetes: results from a British cohort of children and adults. Diabetes Care, 2008. 31(9): pp. 1789-94.

[54] Boyle, M.P., and De Boeck, K., A new era in the treatment of cystic fibrosis: correction of the underlying CFTR defect. Lancet Respir Med, 2013. 1(2): pp. 158-63.

[55] De Boeck, K., et al., The relative frequency of CFTR mutation classes in European patients with cystic fibrosis. J Cyst Fibros, 2014. 13(4): pp. 403-9.

[56] Leung, D.H., et al., Baseline ultrasound and clinical correlates in children with cystic fibrosis. J Pediatr, 2015. 167(4): pp. 862-8 e2.

[57] Finkelstein, S.M., et al., Diabetes mellitus associated with cystic fibrosis. J Pediatr, 1988. 112(3): pp. 373-7.

[58] Moran, A., et al., Cystic fibrosis-related diabetes: current trends in prevalence, incidence, and mortality. Diabetes Care, 2009. 32(9): pp. 1626-31.

[59] Lewis, C., et al., Diabetes-related mortality in adults with cystic fibrosis. Role of genotype and sex. Am J Respir Crit Care Med, 2015. 191(2): pp. 194-200.

[60] Campbell, J., et al., 328 Use of an insulin pump combined with the FreeStyle Libre interstitial glucose monitor in a needle-phobic adolescent with cystic fibrosis-related diabetes. J Cyst Fibros, 2015. 14: p. S142.

[61] Lanng, S., et al., Influence of the development of diabetes mellitus on clinical status in patients with cystic fibrosis. Eur J Pediatr, 1992. 151(9): pp. 684-7.

[62] Brennan, A.L., et al., Airway glucose concentrations and effect on growth of respiratory pathogens in cystic fibrosis. J Cyst Fibros, 2007. 6(2): pp. 101-9.

[63] Philips, B.J., et al., Glucose in bronchial aspirates increases the risk of respiratory MRSA in intubated patients. Thorax, 2005. 60(9): pp. 761-4.

[64] Hameed, S., et al., Early glucose abnormalities in cystic fibrosis are preceded by poor weight gain. Diabetes Care, 2010. 33(2): pp. 221-6.

[65] van den Borst, B., et al., Pulmonary function in diabetes: a metaanalysis. Chest, 2010. 138(2): pp. 393-406.

[66] Mohamad, I.L., et al., Evaluation of pulmonary function changes in children with type 1 diabetes mellitus in Upper Egypt. Therapeutic Adv Endocrinol Metab, 2015: p. 2042018815580514.

[67] Kraemer, R., et al., Relative underweight in cystic fibrosis and its prognostic value. Acta Paediatr Scand, 1978. 67(1): pp. 33-7. 
[68] Moran, A., et al., Diagnosis, screening and management of cystic fibrosis related diabetes mellitus: a consensus conference report. Diabetes Res Clin Pract, 1999. 45(1): pp. 61-73.

[69] Lanng, S., et al., Glucose tolerance in patients with cystic fibrosis: five year prospective study. BMJ, 1995. 311(7006): pp. 655-9.

[70] Bennett, P.H., Burch, T.A., and Miller, M., Diabetes mellitus in American (Pima) Indians. Lancet, 1971. 2(7716): pp. 125-8.

[71] Gilchrist, F.J., et al., Diabetic retinopathy in patients who do not meet the diagnostic criteria for cystic fibrosis related diabetes. Practical Diabetes, 2015. 32(9): pp. 333-335a.

[72] Lanng, S., et al., Diabetes mellitus in cystic fibrosis: effect of insulin therapy on lung function and infections. Acta Paediatr, 1994. 83(8): pp. 849-53.

[73] Hameed, S., et al., Once daily insulin detemir in cystic fibrosis with insulin deficiency. Arch Dis Child, 2012. 97(5): pp. 464-7.

[74] Widger, J., Ranganathan, S., and Robinson, P.J., Progression of structural lung disease on CT scans in children with cystic fibrosis related diabetes. J Cyst Fibros, 2013. 12(3): pp. 216-21.

[75] Schmid, K., et al., Predictors for future cystic fibrosis-related diabetes by oral glucose tolerance test. J Cyst Fibros, 2014. 13(1): pp. 80-5.

[76] Brodsky, J., et al., Elevation of 1-hour plasma glucose during oral glucose tolerance testing is associated with worse pulmonary function in cystic fibrosis. Diabetes Care, 2011. 34(2): pp. 292-5.

[77] Coriati, A., et al., Characterization of patients with cystic fibrosis presenting an indeterminate glucose tolerance (INDET). J Cyst Fibros, 2016. 15(1): pp. 127-32.

[78] Hameed, S., Jaffe, A., and Verge, C.F., Cystic fibrosis related diabetes (CFRD)--the end stage of progressive insulin deficiency. Pediatr Pulmonol, 2011. 46(8): pp. 747-60.

[79] O'Riordan, S.M., et al., Validation of continuous glucose monitoring in children and adolescents with cystic fibrosis: a prospective cohort study. Diabetes Care, 2009. 32(6): pp. 1020-2.

[80] Dobson, L., Sheldon, C.D., and Hattersley, A.T., Validation of interstitial fluid continuous glucose monitoring in cystic fibrosis. Diabetes Care, 2003. 26(6): pp. 1940-1.

[81] Bergenstal, R.M., et al., Effectiveness of sensor-augmented insulin-pump therapy in type 1 diabetes. N Engl J Med, 2010. 363(4): pp. 311-20.

[82] Moreau, F., et al., Continuous glucose monitoring in cystic fibrosis patients according to the glucose tolerance. Horm Metab Res, 2008. 40(7): pp. 502-6.

[83] Schiaffini, R., et al., Abnormal glucose tolerance in children with cystic fibrosis: the predictive role of continuous glucose monitoring system. Eur J Endocrinol, 2010. 162(4): pp. 705-10.

[84] Bennett, C., Guo, M., and Dharmage, S., HbA1c as a screening tool for detection of type 2 diabetes: a systematic review. Diabet Med, 2007. 24(4): pp. 333-43. 
[85] Leclercq, A., et al., Early assessment of glucose abnormalities during continuous glucose monitoring associated with lung function impairment in cystic fibrosis patients. J Cyst Fibros, 2014. 13(4): pp. 478-84.

[86] Moran, A., et al., Epidemiology, pathophysiology, and prognostic implications of cystic fibrosisrelated diabetes: a technical review. Diabetes Care, 2010. 33(12): pp. 2677-83.

[87] Hardin, D.S., et al., Use of the insulin pump in treat cystic fibrosis related diabetes. J Cyst Fibros, 2009. 8(3): pp. 174-8.

[88] Thabit, H., et al., Home use of an artificial beta cell in type 1 Diabetes. N Engl J Med, 2015. 373(22): pp. 2129-40.

[89] Stallings, V.A., et al., Evidence-based practice recommendations for nutrition-related management of children and adults with cystic fibrosis and pancreatic insufficiency: results of a systematic review. J Am Diet Assoc, 2008. 108(5): pp. 832-9.

[90] Wilson, D., et al., Challenges in the dietary treatment of cystic fibrosis related diabetes mellitus. Clin Nutr, 2000. 19(2): pp. 87-93.

[91] Colombo, C., et al., Liver disease in cystic fibrosis: a prospective study on incidence, risk factors, and outcome. Hepatology, 2002. 36(6): pp. 1374-82. 